\title{
INFLUÊNCIA DO PH NA DEGRADAÇÃO DE SULFAMETOXAZOL E TRIMETOPRIMA POR PROCESSO FOTO-FENTON SOLAR MEDIADO POR FERRIOXALATO
}

\author{
I. N. DIAS ${ }^{1,3}$, B. SOUZA ${ }^{2}$, V. J. P. VILAR ${ }^{3}$, M. DEZOTTI ${ }^{1}$ \\ ${ }^{1}$ Universidade Federal do Rio de Janeiro, Programa de Engenharia Química \\ ${ }^{2}$ Universidade Federal de Sergipe , Núcleo de Engenharia Ambiental \\ ${ }^{3}$ Faculdade de Engenharia da Universidade do Porto, Departamento de Engenharia Química \\ E-mail para contato: isabelli@peq.coppe.ufrj.br
}

RESUMO - Antibióticos como sulfametoxazol (SMX) e trimetoprima (TMP), são lançados em corpos hídricos e, devido à ineficiência do tratamento convencional, buscamse tecnologias mais avançadas para remoção desses compostos. Neste trabalho, a redução de SMX e TMP (20 mg/L) foi avaliada por processo foto-Fenton solar, utilizando ferrioxalato. A concentração de ferro foi $5 \mathrm{mg} / \mathrm{L} \mathrm{e} \mathrm{o} \mathrm{pH}$ variou de 4,0 a 6,5. Observou-se que a degradação dos antibióticos diminuiu com o aumento do $\mathrm{pH}$ devido a diminuição do complexo mais fotoativo, mas, o processo foi capaz de degradar SMX e TMP em pH próximo da neutralidade. Além disso, apesar da eficiência de degradação dos antibióticos, a pH 4,0 ter sido muito semelhante, a degradação de SMX foi mais afetada pelo aumento do $\mathrm{pH}$, uma vez que as principais espécies de SMX presente em solução são neutras ou carregadas negativamente, podendo formar complexos estáveis com as espécies de ferro, limitando a foto-redução de $\mathrm{Fe}^{3+}$, diminuindo a eficiência do processo.

\section{INTRODUÇÃO}

A presença de micropoluentes em corpos hídricos constitui hoje uma grande preocupação ambiental (Richardson, 2006; Le-Minh et al., 2010). Os antibióticos merecem uma atenção especial devido à sua ampla utilização e persistência no meio ambiente, mesmo em concentrações vestigiais. $\mathrm{O}$ sulfametoxazol (SMX) e trimetoprima (TMP) são antibióticos muito utilizados, sendo que os resíduos desses compostos são lançados nos corpos d'água de diferentes formas: através de despejos industriais (indústria farmacêutica), esgoto doméstico e hospitalar. Esses compostos são frequentemente detectados no efluente final de instalações de tratamento, mostrando que eles não são eliminados durante o processo biológico convencional, podendo ocasionar o crescimento de microrganismos resistentes. Assim, os processos oxidativos avançados (POA) consistem em um método alternativo e eficiente na degradação de fármacos. Os POA baseiam-se na geração de espécies químicas altamente reativas como $\bullet \mathrm{OH}$ (Homem e Santos, 2011). Um dos POA amplamente utilizado na degradação de micropoluentes é o processo foto-Fenton, no entanto, as maiores limitações desse processo aplicado ao tratamento dos efluentes finais estão associadas à necessidade de acidificação, a fim de evitar a precipitação de ferro, e a necessidade de remoção de ferro a fim de obedecer aos limites de descarga. Uma maneira de tornar a oxidação por processo foto-Fenton mais atraente e conduzi-la em valor de 
pH neutro é alterando a fonte fotocatalisador .

O uso de complexos, tais como ferrioxalato, que são mais solúveis, permite trabalhar a valores de pH neutros, evitando as necessidades de acidificação e neutralização. Além disso, estes complexos têm i) rendimentos quânticos muito mais elevados do que os complexos de ferro e água; ii) podem usar uma fração superior do espectro da radiação solar, até $580 \mathrm{~nm}$; iii) são fotodescarboxilados sob radiação visível; e iv) proporcionam uma via mais rápida para regeneração de $\mathrm{Fe}^{3+}$, acelerando assim o processo.

O presente estudo avaliou a eficiência de degradação de soluções aquosas individuais de SMX e TMP, com uma concentração inicial de $20 \mathrm{mg} / \mathrm{L}$, por um processo foto -Fenton solar, próximo da neutralidade, utilizando complexo de ferro. Em particular, o uso de ácido de oxálico como um agente quelante para o ferro aumenta a atividade fotocatalítica do sistema foto-Fenton, permitindo que a reação ocorra em pH próximo da neutralidade (Cheng et al., 2011).

\section{MATERIAIS E MÉTODOS}

Os experimentos de foto-Fenton foram realizados em um foto-reator em escala laboratorial com um simulador de luz solar, instalado no Departamento de Engenharia Química (DEQ) da Faculdade de Engenharia da Universidade do Porto (FEUP). O sistema fotocatalítico é composto por (Figura 1): i) um simulador de radiação solar (ATLAS, modelo SUNTEST XLS+) com $1100 \mathrm{~cm}^{2}$ de área de exposição, uma lâmpada de xenônio de 1700 Watts, um filtro de luz solar e um filtro de quartzo; ii) um coletor parabólico composto (CPC) $\operatorname{com} 0,023 \mathrm{~m}^{2}$ de área iluminada com refletor de alumínio anodizado e tubo de borossilicato (Schott-Duran, tipo 3.3, cut-off $280 \mathrm{~nm}$, diâmetro interno 46,4 mm, comprimento $160 \mathrm{~mm}$, largura $1,8 \mathrm{~mm}$ ); iii) um recipiente de vidro (capacidade de 1,5 litros) encamisado acoplado a um banho termostático (Lab. Companion, modelo RW-0525G) para o controle da temperatura durante a reação; iv) um agitador magnético (Velp Scientifica , modelo ARE) para assegurar a homogeneização completa da solução no interior do recipiente de vidro; v) uma bomba peristáltica (Ismatec, modelo Ecoline VC-380 II) para promover a recirculação de água entre o CPC e o recipiente de vidro; vi) medidor de $\mathrm{pH}$ e temperatura (VWR symphony - SB90M5). A radiação foi medida através de um radiômetro UV de banda larga (Kipp \& Zonen B.V., modelo CUV5) que foi colocado no interior do reator solar no mesmo nível do centro do fotoreator.

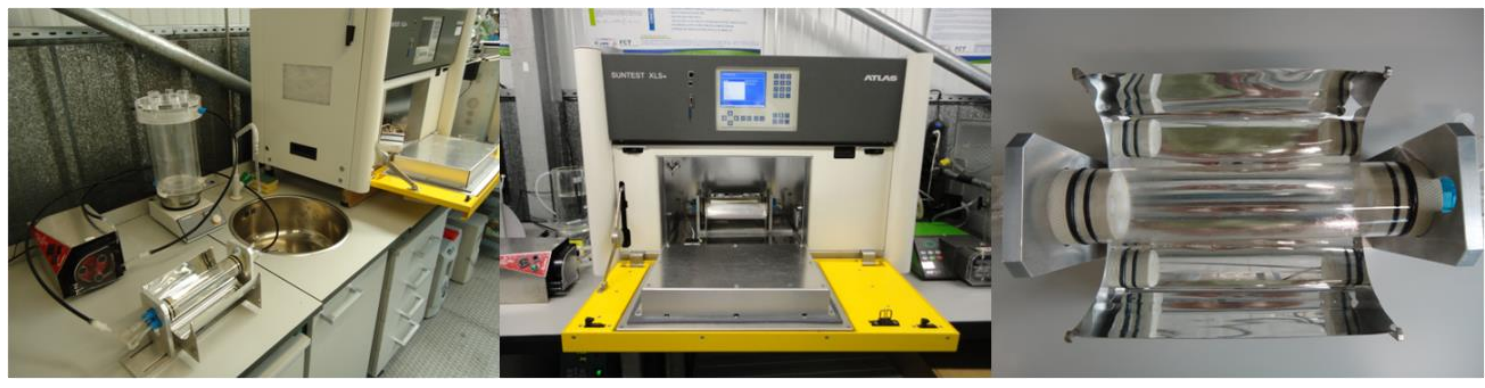

Figura 1 - Fotoreator com simulador de luz solar (SUNTEST). 


\section{9 a 22 de outubro de 2014 \\ Florianópolis/SC}

A fim de avaliar a reação de foto-Fenton modificada na degradação dos fármacos, utilizou-se 1,3 L da solução de antibiótico $(20 \mathrm{mg} / \mathrm{L})$. A solução foi bombeada para a unidade de CPC e homogeneizada por recirculação no sistema fechado durante 15 minutos, no escuro. Em seguida, foi adicionado ácido oxálico, (razão molar: $1 \mathrm{Fe}^{3+}: 3$ oxalato $\left(\mathrm{C}_{2} \mathrm{O}_{4}{ }^{-2}\right)$ ), com o qual o pH foi ajustado $(4,0$, 4,5, 5,0 ou 5,5). Amostras foram retiradas após homogeneização da solução e correção do pH. Cloreto férrico foi adicionado para se obter as concentrações de 5,0 $\mathrm{mg} \mathrm{Fe}^{3+} / \mathrm{L}$. Após $15 \mathrm{~min}$, foi feita mais uma amostragem para controle da concentração de ferro. O banho termostático foi utilizado para controlar e manter a temperatura em $25{ }^{\circ} \mathrm{C}$. O SUNTEST foi ligado e a intensidade da radiação foi definida como $500 \mathrm{~W} / \mathrm{m}^{2}$, o que é equivalente a $44 \mathrm{~W}$ Uv $/ \mathrm{m}^{2}$, medida na gama de comprimentos de onda de 280-400 nm. Finalmente, uma dose de peróxido de hidrogênio foi adicionada, equivalente à quantidade estequiométrica necessária para mineralizar completamente a solução de antibiótico. $\mathrm{O}$ pH foi controlado durante todas as reações. Em todos os casos, as amostras foram colhidas em intervalos de tempo sucessivos para avaliar o progresso da reação foto-Fenton modificada.

A eficiência do processo foi avaliada em termos de remoção de carbono orgânico dissolvido (COD), medido em um analisador de carbono orgânico total Shimadzu, modelo TOC- $\mathrm{V}_{\mathrm{CSN}}$, seguindo os métodos 5310 A e B padronizados (APHA, 2005) e degradação do antibiótico, utilizando um cromatógrafo líquidoVWR Hitachi LaChrom ELITE HPLC (Merck-Hitach, Tóquio, Japão) com coluna de fase reversa Purospher $®$ RP-18e, 125 x 4mm (5 um) (Merck). Os limites de quantificação e detecção foram 0,079/0,024 e 0,013/0,004 $\mathrm{mg} \mathrm{L}^{-1}$ para TMP e SMX, respectivamente.

\section{RESULTADOS E DISCUSSÕES}

O pH ótimo para a reação de foto-Fenton é de 2,8, não só porque a espécie predominante de ferro em solução é $\mathrm{FeOH}^{2+}$, que é o complexo de íon férrico em água mais fotoativo, mas também porque se evita a precipitação de ferro, No entanto, as maiores limitações do processo foto-Fenton estão associadas à necessidade de acidificação e posterior neutralizaçao. $\mathrm{O}$ uso de complexos, tais como ferrioxalato, permite trabalhar a valores de $\mathrm{pH}$ próximo da neutralidade, evitando a necessidade de acidificação.

A Figura 2 apresenta os perfis de degradação e mineralização dos antibióticos para o processo foto-Fenton mediado por ferrioxalato em diferentes valores de $\mathrm{pH}$, a temperatura constante $\left(25^{\circ} \mathrm{C}\right) \mathrm{e}$ concentração de ferro de $5 \mathrm{mg} \mathrm{Fe} \mathrm{Fe}^{3+} / \mathrm{L}$. É importante mencionar que o aumento súbito inicial no valor de COD está relacionado a adição de ácido oxálico (cerca de $5 \mathrm{mg} \mathrm{C/L}$ ). Pode ser observado que a taxa de degradação de ambos os antibióticos diminui com o aumento do pH de trabalho, de modo que, a remoção completa de antibióticos, tendo em vista o limite de detecção do método analítico, só foi alcançada após uma dose de energia UV acumulada de 0,24/0,24 kJ/L, 1,0/0,25 kJ/L, 3,1/0,5 kJ/L e 5,0/1,0 kJ/L para soluções de SMX/TMP em pH 4,0, 4,5, 5,0 e 5,5, respectivamente. 


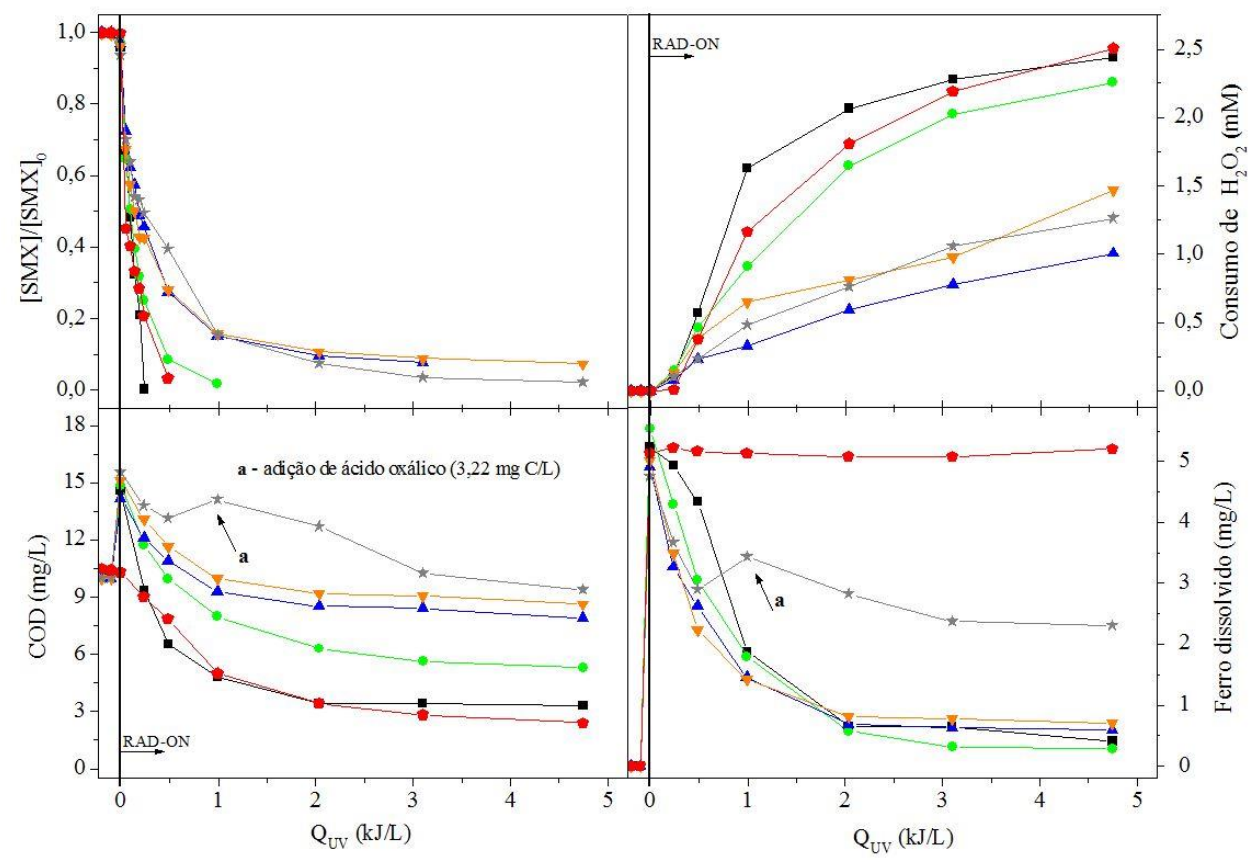

(a)

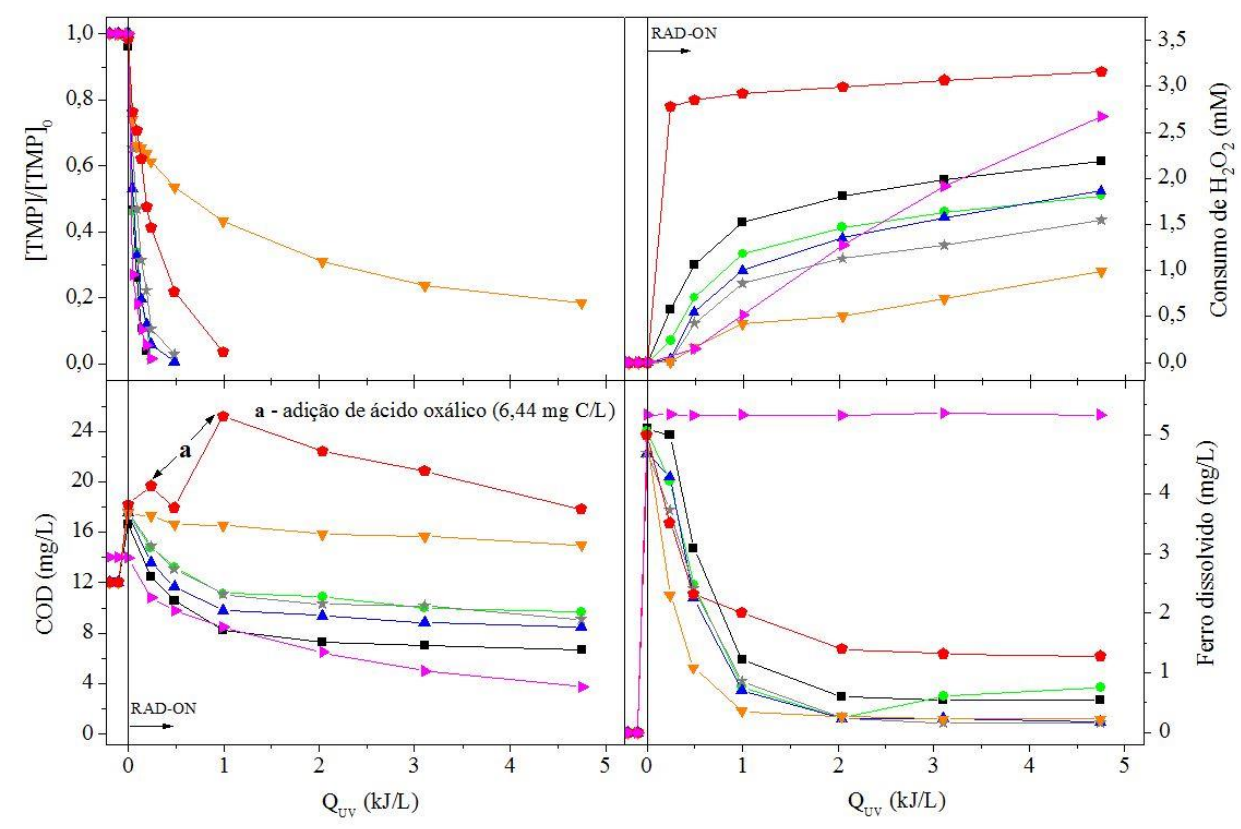

(b)

Figura 2 - Efeito do pH na degradação as soluções $\left(C_{0}=20 \mathrm{mg} / \mathrm{L}\right)$ de SMX (a) e TMP (b) usando processo foto-Fenton solar mediado por complexo ferrioxalato (razão molar de ferro: oxalato de 1:3) $\left(\mathrm{Fe}^{3+} / \mathrm{H}_{2} \mathrm{O}_{2}\right.$ /ácido oxálico/UV): (a) SMX: ( ) - $\mathrm{pH}$ 2,8 (foto-Fenton clássico com 5,0 $\mathrm{mg} \mathrm{Fe}^{2+} / \mathrm{L}$ );

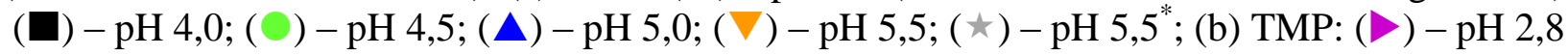




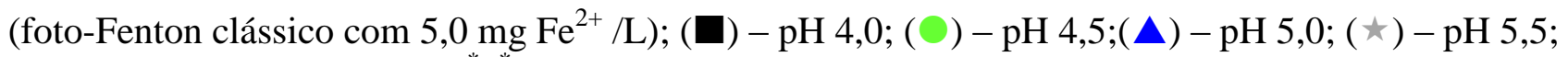

$(\nabla)-\mathrm{pH} 6,5 ;(\bullet)-\mathrm{pH} 6,5^{*}$. ${ }^{*}$ Experimento realizado com adição de ácido oxálico em diferentes tempos de reação. Condições de processos: $T=25^{\circ} \mathrm{C},\left[\mathrm{Fe}^{3+}\right] \sim 5 \mathrm{mg} / \mathrm{L}$.

Segundo Faust e colaboradores (1993), o complexo $\mathrm{Fe}\left(\mathrm{C}_{2} \mathrm{O}_{4}\right)_{2}{ }^{-}$tem um rendimento quântico mais elevado $(1,0)$ para a formação de $\mathrm{Fe}^{2+}$ (regeneração do ferro) do que o complexo $\mathrm{Fe}\left(\mathrm{C}_{2} \mathrm{O}_{4}\right)_{3}{ }^{3-}$, que apresenta rendimento de 0,6 a $436 \mathrm{~nm}$. Considerando-se o diagrama de especiação (Figura 3), a fração molar de $\mathrm{Fe}\left(\mathrm{C}_{2} \mathrm{O}_{4}\right)_{2}{ }^{-}$diminuiu de $61,3 \mathrm{em} \mathrm{pH} \mathrm{4,0} \mathrm{para} \mathrm{30,8 \%} \mathrm{em} \mathrm{pH}$ 6,5. Por outro lado, a fração molar de $\mathrm{Fe}\left(\mathrm{C}_{2} \mathrm{O}_{4}\right)_{3}{ }^{3-}$ aumentou de $37,8 \%$ em $\mathrm{pH} 4,0$ para 55,1\% em $\mathrm{pH} 6,5$, o que pode explicar a menor eficiência da reação foto-Fenton na degradação do antibiótico para valores de $\mathrm{pH}$ mais elevados .

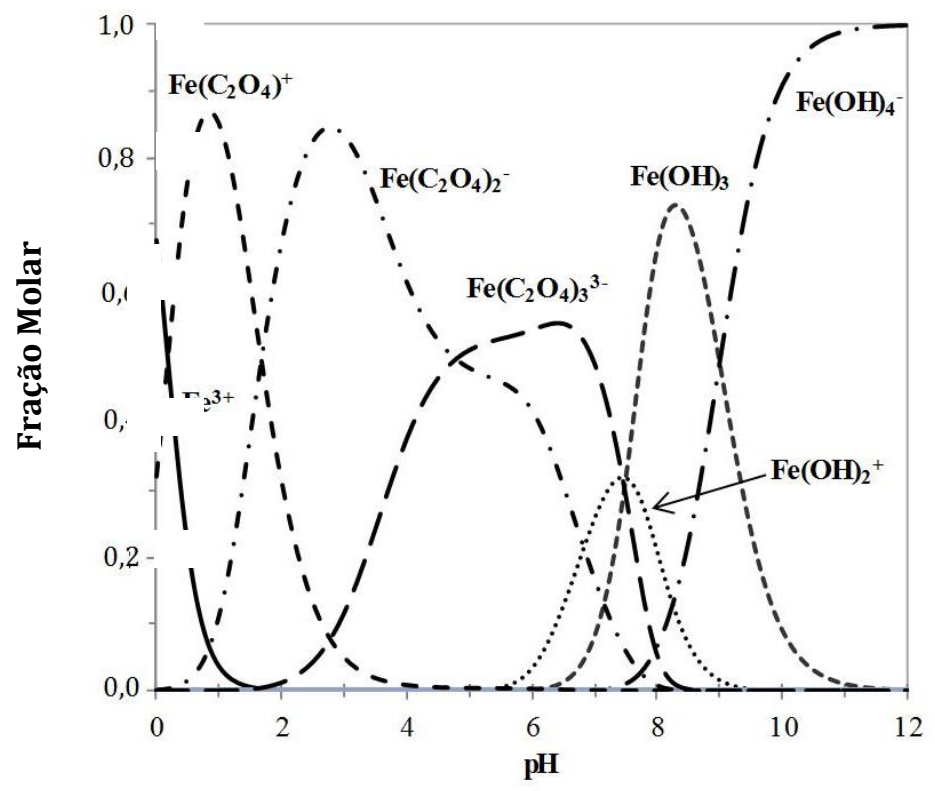

Figura 3 - Diagrama de especiação dos complexos ferri-oxálicos em função do pH para uma solução contendo $2,69 \times 10^{-1} \mathrm{mM}(24,2 \mathrm{mg} / \mathrm{L})$ de ácido oxálico e $8,95 \times 10^{-2} \mathrm{mM}(5 \mathrm{mg} / \mathrm{L})$ de ferro (Balmer \& Sulzberger, 1999).

Além disso, comparando, na Figura 2, os perfis de degradação entre o processo foto-Fenton clássico $(\mathrm{pH}$ 2,8) e o processo de foto-Fenton mediado por ferrioxalato, para a mesma concentração de ferro (5,0 mg de $\mathrm{Fe}^{2+}$ ou $\mathrm{Fe}^{3+} \mathrm{L}^{-1}$ ), observou-se que a degradação de SMX e TMP obtidos a pH 2,8 para o processo clássico foi semelhante a degradação obtida para o processo com ferrioxalato a $\mathrm{pH}$ 4,0 (SMX) e 5,0 (TMP). Esse resultado pode ser atribuído a presença de ácido oxálico e a consequente formação de complexos fortes, que são mais solúveis do que os complexos hidróxidos férricos.

Verificou-se, ainda, que embora a eficiência de degradação para ambos os antibióticos a pH 4,0 tenha sido muito semelhante, a degradação de SMX foi muito mais afetada pelo aumento 
do $\mathrm{pH}$ da solução do que a de TMP. De acordo com o diagrama de especiação dos antibióticos (Figura 4), para valores de $\mathrm{pH}$ superiores a 4,0 a fração molar da espécie SMX carregada negativamente aumenta significativamente, sendo que esta espécie foi avaliada como a forma mais estável (Niu et al., 2013). Por outro lado, de acordo com o diagrama de especiação do TMP, a espécie predominante na gama de $\mathrm{pH}$ estudada foi a espécie protonada $\left(\mathrm{HTMP}^{+}\right)$e esta espécie apresenta maior reatividade com os radicais hidroxila (González et al., 2011).
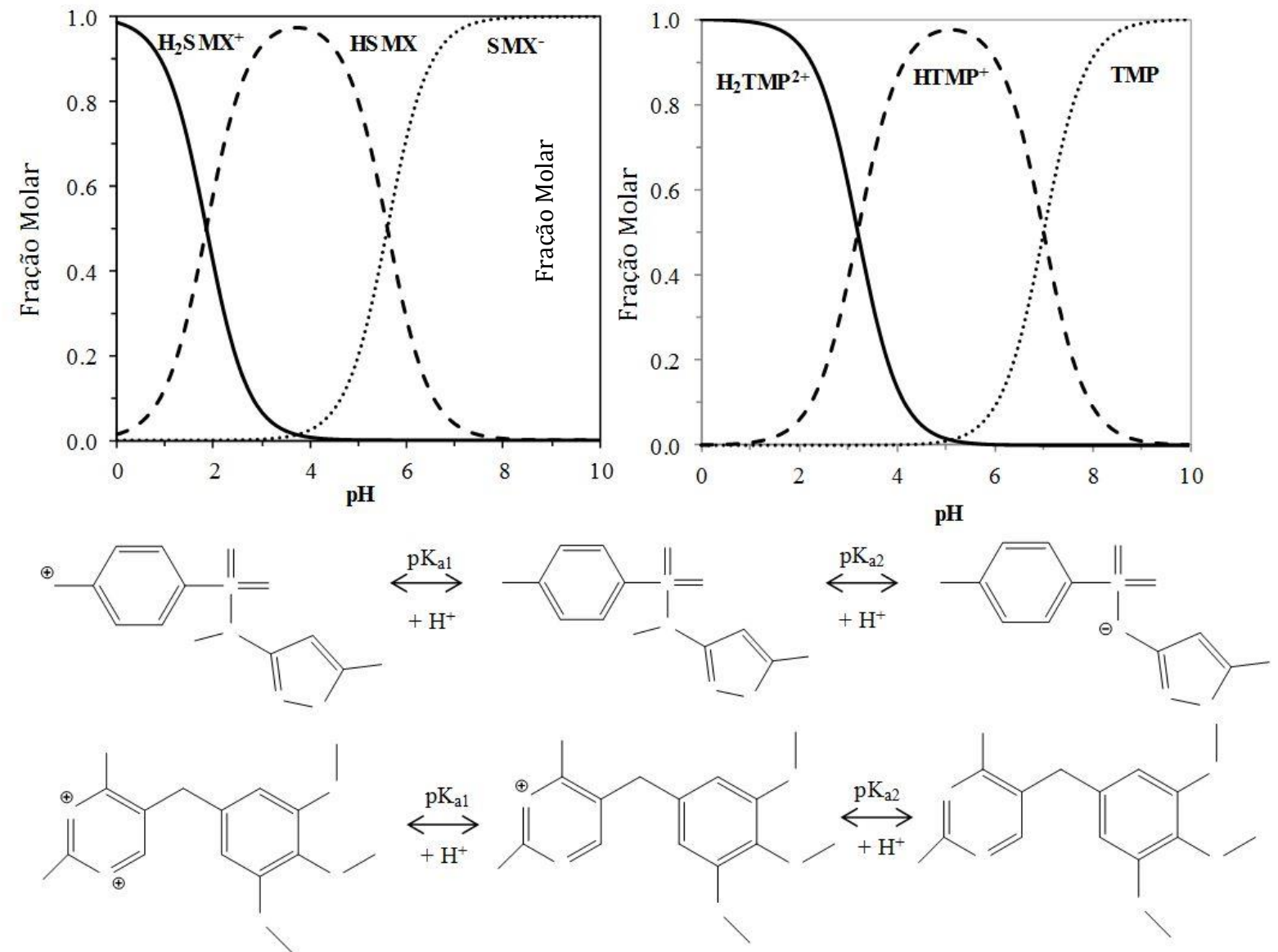

Figura 4 - Diagrama de especiação e estrutura química dos antibióticos Sulfametoxazol (SMX) e Trimetoprima (TMP) (Adaptado de Lin et al., 1997; Qiang \& Adams, 2004).

Dois experimentos adicionais foram realizados fazendo-se mais de uma adição de ácido oxálico ao longo da reação para SMX a pH 5,5 e TMP a pH 6,5. Embora a adição extra de ácido oxálico tenha evitado a precipitação de ferro, aumentando a degradação do antibiótico, principalmente para TMP, a mineralização foi substancialmente reduzida devido à adição de ácido oxálico como fonte de carbono orgânico. 


\section{9 a 22 de outubro de 2014 \\ Florianópolis/SC}

\section{CONCLUSÕES}

A reação foto-Fenton mediada por ferrioxalato foi eficiente na degradação dos antibióticos SMX e TMP em solução aquosas mesmo em condições de $\mathrm{pH}$ neutro e baixa concentração de ferro. A eficiência de degradação dos antibióticos diminuiu significativamente com o aumento do $\mathrm{pH}$, o que está associada a diminuição da espécie de complexo ferrioxalato mais fotoativo, alem da precipitação de ferro, devido ao consumo de ácido oxálico. As espécies do antibiótico em solução afeta fortemente a eficiência da degradação, principalmente devido aos complexos formados entre as espécies de ferro carregados positivamente e as espécies carregadas negativamente antibióticos.

\section{AGRADECIMENTOS}

Esse trabalho foi parcialmente financiado pelos projetos PEst-C/EQB/LA0020/2011 e PTDC/AAC-AMB/113091/2009, FCT - Fundação para a Ciência e a Tecnologia. CAPES/FCT 308/11 (Concessão de pesquisa- Processo BEX: 8989/11-7) e FAPERJ (Processo n. ${ }^{\circ}$ 100.268/2013).

\section{REFERÊNCIAS}

APHA, AWWA, WEF. Standard Methods for the Examination of Water and Wastewater, 21th edition, American Public Health Association, American Water Works Association, Water Pollution Control Federation, Washington DC, USA, 2005.

BALMER, M. E.; SULZBERGER, B. Atrazine Degradation in Irradiated Iron/Oxalate Systems: Effects of pH and Oxalate. Environmental Science and Technology, v. 33, pp. 2418-2424, 1999.

CHENG, H. P.; HUANG, Y. H.; LEE, C. Decolorization of reactive dye using a photo-ferrioxalate system with brick grain-supported iron oxide. Journal of Hazardous Materials, v. 188, pp. 357-362, 2011.

FAUST, B. C.; ZEPP, R. G. Photochemistry of aqueous iron(III)-polycarboxylate complexes: roles in the chemistry of atmospheric and surface waters. Environmental Science \& Technology, v. 27, pp. 2517-2522, 1993.

GONZÁLEZ, T.; DOMÍNGUEZ, J. R.; PALO, P.; et al. Development and Optimization of the BDDElectrochemical Oxidation of the Antibiotic Trimethoprim in Aqueous Solution, Desalination, v. 280 , pp. 197-202, 2011.

HOMEM, V.; SANTOS, L. Degradation and removal methods of antibiotics from aqueous matrices. A review. Journal of Environmental Management, v. 92, pp. 2304-2347, 2011.

LE-MINH, N.; KHAN, S.; DREWES, J.; STUETZ, R. Fate of antibiotics during municipal water recycling treatment processes. Water Research, v. 44, pp. 4295-4323, 2010. 
LIN, C. E.; CHANG, C. C.; LIN, W. C. Migration Behavior and Separation of Sulfonamides in Capillary Zone Electrophoresis: III. Citrate Buffer as a Background Electrolyte, Journal of Chromatography A, v. 768, pp. 105-112, 1997.

NIU, J.; ZHANG, L.; LI, Y.; et al. Effects of Environmental Factors on Sulfamethoxazole Photodegradation Under Simulated Sunlight Irradiation: Kinetics and Mechanism, Journal of Environmental Sciences, v. 25, pp. 1098-1106, 2013.

QIANG, Z.; ADAMS, C.; Potentiometric Determination of Acid Dissociation Constants (pKa) for Human and Veterinary Antibiotics, Water Research, v. 38, pp. 2874-2890, 2004.

RICHARDSON, S.D. Environmental mass spectrometry: emerging contaminants and current issues. Analytical Chemistry-Columbus, v. 78, pp. 4021-4046, 2006. 